\title{
OBSTRUCTION THEORIES FOR SMOOTHING MANIFOLDS AND MAPS
}

\author{
BY MORRIS W. HIRSCH
}

\author{
Communicated by Deane Montgomery, January 24, 1963
}

1. Introduction. In his papers [5] and [6], James Munkres defines two obstruction theories. The first attacks the problem of smoothing a map, the second that of smoothing a manifold. We present two similar obstruction theories which avoid certain difficulties present in the earlier ones.

The obstruction cochains are defined and their properties stated in $\$ \S 3$ and 4 . $\$ 2$ presents the fundamental result on which the paper is based. In $\$ 5$ we outline a proof of the conjecture of John Milnor that $\Gamma_{i-1}=\pi_{i}\left(B_{P L}, B_{O}\right)$. Details will be presented elsewhere.

Some of these results have been obtained independently by Barry Mazur.

2. The Product Theorem. A differential manifold will be denoted by an ordered pair $M_{\alpha}$, where $M$ is a combinatorial manifold and $\alpha$ is a compatible differential structure on $M$. (Strictly speaking, $M_{\alpha}$ is a differential manifold with a distinguished class of smooth triangulations.) If $U \subset M$ is an open set, then $\alpha \mid U$ and $U_{\alpha}$ have the obvious meanings. We call $\alpha$ a smoothing of $M$.

Let $I$ be the closed unit interval. Two smoothings $\alpha, \beta$ of an unbounded combinatorial manifold $M$ are concordant if there is a smoothing $\gamma$ of $M \times I$ such that the boundary of $(M \times I)_{\gamma}$ is $(M \times 0)_{\alpha}$ $\cup(M \times 1)_{\beta}$. (This definition is due to Milnor.)

Suppose that there is a smoothing $\delta$ of a neighborhood $U$ of a subcomplex $K \subset M$ such that $(U \times I)_{\gamma}=U_{\delta} \times I$. Then we say that $\alpha$ and $\beta$ are concordant rel $K$; the notation is $M_{\alpha} \bumpeq M_{\beta}$ rel $K$.

Concerning the relationship between concordance and diffeomorphism, the following results are known:

Theorem 1.1. (a) Concordance implies diffeomorphism (Thom [8], Munkres [7]).

(b) For spheres, diffeomorphism implies concordance (Milnor).

(c) There are smooth manifolds which are diffeomorphic but not concordant.

For example, let $\alpha, \beta$ be smoothings of the combinatorial $n$-sphere $S$ such that $S_{\alpha}$ and $S_{\beta}$ are not concordant. It is known [4] that for large enough $m, S_{\alpha} \times R^{m}$ and $S_{\beta} \times R^{m}$ are diffeomorphic. However, it 
follows from the Product Theorem that if $S_{\alpha} \times R^{m} \bumpeq S_{\beta} \times R^{m}$, then $S_{\alpha} \bumpeq S_{\beta}$.

Theorem 2.1 (Product Theorem). Let $\alpha$ be a smoothing of $a$ neighborhood $U$ of a subcomplex $K$ of a combinatorial unbounded manifold $M ; K$ may be empty. Let $\beta$ be a smoothing of $M \times R^{n}$ such that $\left(U \times R^{n}\right)_{\beta}=U_{\alpha} \times R^{n}$. There is a smoothing $\gamma$ of $M$ with the following properties:

(a) $M_{\gamma} \times R^{n} \bumpeq\left(M \times R^{n}\right)_{\beta}$ rel $K \times R^{n}$.

(b) There is a neighborhood $V$ of $K$ in $U$ such that $\gamma|V=\alpha| V$. Moreover, any two smoothings of $M$ satisfying (a) and (b) are concordant rel $K$.

The proof of existence is by induction on $n$. The case $n=1$ is essentially contained in Theorem 2.5 of [2]. The induction is completed by observing that $M \times R^{n+1}=\left(M \times R^{n}\right) \times R^{1}$. Uniqueness follows easily from existence.

3. Obstructions to smoothing manifolds. Recall that $\Gamma_{k}$ may be defined as the group of oriented diffeomorphism classes of smoothings of the combinatorial $k$-sphere $S^{k}$. The group operation is the formation of the connected sum.

Let $M$ be a combinatorial $n$-manifold, with a fixed triangulation. Let $K$ be a subcomplex. Denote the $i$-skeleton of $M$ by $M_{i}$. Let $\alpha$ be a smoothing of a neighborhood $U$ of $K \subset M_{i}$, and let $\sigma^{i+1}$ be an $(i+1)$ simplex of $M$ with boundary $\partial \sigma^{i+1}$. There is an open regular neighborhood $N\left(\partial \sigma^{i+1}\right)$ inside $U$. Now $N\left(\partial \sigma^{i+1}\right)$ and $S^{i} \times R^{n-i}$ are combinatorially equivalent, denoted by $N\left(\partial \sigma^{i+1}\right) \equiv S^{i} \times R^{n-i}$. From the Product Theorem (2.1) it follows that there is a unique element $\beta \in \Gamma_{i}$ such that $N\left(\partial \sigma^{i+1}\right)_{\alpha} \bumpeq\left(\partial \sigma^{i+1}\right)_{\beta} \times R^{n-i}$. Put $\beta=C_{\alpha}\left(\sigma^{i+1}\right)$.

Theorem 3.1. The cochain $C_{\alpha} \in C^{i+1}\left(M ; \Gamma_{i}\right)$ has the following properties:

(a) $C_{\alpha}$ is a cocycle and vanishes on simplices in $K$.

(b) $C_{\alpha}\left(\sigma^{i+1}\right)=0$ if and only if the smoothing $\alpha$ can be extended over a neighborhood of $\sigma^{i+1}$.

(c) If $\alpha^{\prime}$ is a smoothing of a neighborhood of $K \cup M_{i}$ which agrees with $\alpha$ in a neighborhood of $K \cup M_{i-1}$, then $C_{\alpha^{\prime}}-C_{\alpha}$ is a coboundary $\bmod K$, and every $(i+1)$-coboundary $\bmod K$ is obtained as $\alpha^{\prime}$ varies.

(d) If $\alpha^{\prime}$ is a smoothing of a neighborhood of $K \cup M_{i}$ such that $\alpha^{\prime} \bumpeq \alpha$, then $C_{\alpha^{\prime}}=C_{\alpha}$.

4. Obstructions to smoothing maps. Let $M$ be a combinatorial manifold and $N$ a differential manifold. A homeomorphism $f: M \rightarrow N$ is piecewise regular if each closed simplex of some rectilinear triangu- 
lation of $M$ is mapped diffeomorphically. Two such maps $f_{i}: M \rightarrow N$ $(i=0,1)$ are concordant rel $K$ (where $K \subset M$ is a subcomplex) if there is a piecewise regular homeomorphism $G: M \times I \rightarrow N \times I$ such that for $i=0,1$ we have $G(x, i)=\left(f_{i}(x), i\right)$, and such that for some neighborhood $W$ of $K \times I$ in $M \times I$, we have $G(x, t)=\left(f_{0}(x), t\right)$ for all $(x, t) \in W$.

The following result is a strengthening of $1.1 \mathrm{a}$.

THEOREM 4.1. Let $\alpha, \beta$ be smoothings of $M$ that are concordant rel $K$. Then there is a diffeomorphism $g: M_{\alpha} \rightarrow M_{\beta}$ that is concordant rel $K$ to the identity map $M_{\alpha} \rightarrow M_{\beta}$.

This theorem translates the problem of concordance of maps into a problem of concordance of differential structures, as follows. Let $f: M_{\alpha} \rightarrow V_{\beta}$ be a piecewise regular homeomorphism. Let $K \subset M$ be a subcomplex, and suppose that $f$ maps a neighborhood of $K$ diffeomorphically; we say $f$ is smooth near $K$. Suppose in particular that $f$ is smooth near $K \cup M_{i-1}$. We ask for a piecewise regular homeomorphism $g: M_{\alpha} \rightarrow V_{\beta}$ which is smooth near $K \cup M_{i}$, and which is concordant to $f$ rel $K \cup M_{i-1}$. It follows from Theorem 4.1 that this is the case if and only if there is a smoothing $\gamma$ of $M \times R$ such that $(M \times R)_{\gamma}$ agrees with $M_{\alpha} \times R$ in a neighborhood of $M \times(-\infty, 0]$ $\cup\left(K \cup M_{i-1}\right) \times R$ and with $M_{f^{*} \beta}$ in a neighborhood of $M \times[1, \infty)$. (Here $f^{*} \beta$ is the unique smoothing of $M$ such that $f: M_{f^{*} \beta} \rightarrow V_{\beta}$ is a diffeomorphism.)

To define an obstruction cochain, let $\sigma^{i} \subset M$ be an $i$-simplex. The smoothing $\gamma$ described above already exists in a neighborhood of $\sigma^{i} \times 0 \cup \sigma^{i} \times 1 \cup \partial \sigma^{i} \times I=\partial\left(\sigma^{i} \times I\right)$. The obstruction to extending $\gamma$ over a neighborhood of $\sigma^{i} \times I$ lies in $H^{i+1}\left(\sigma^{i} \times I, \partial\left(\sigma^{i} \times I\right) ; \Gamma_{i}\right) \approx \Gamma_{i}$, as described in $\$ 3$. Let this obstruction be denoted by $C_{f}\left(\sigma^{i}\right)$. Thus $C_{f} \in C^{i}\left(M ; \Gamma_{i}\right)$.

TheOREM 4.2. The cochain $C_{f}$ has the following properties:

(a) $C_{f}$ is a cocycle and vanishes on simplices in $K$.

(b) $C_{f}\left(\sigma^{i}\right)=0$ if and only if $f$ is concordant rel $K \cup M_{i-1}$ to a piecewise regular homeomorphism $g: M_{\alpha} \rightarrow V_{\beta}$ which is smooth near $K \cup M_{i-1}$ $\cup \sigma^{i}$

(c) If $f^{\prime}: M_{\alpha} \rightarrow V_{\beta}$ is a piecewise regular homeomorphism which agrees with $f$ in a neighborhood of $K \cup M_{i-2}$, then $C_{f^{\prime}}-C_{f}$ is a coboundary $\bmod K$, and every coboundary $\bmod K$ is obtained as $f^{\prime}$ varies.

(d) If $f^{\prime}: M_{\alpha} \rightarrow V_{\beta}$ is a piecewise regular homeomorphism which is smooth near $K \cup M_{i-1}$, and $f^{\prime}$ is concordant to $f$ by a piecewise regular 
homeomorphism $G: M_{\alpha} \times I \rightarrow V_{\beta} \times I$ which is smooth near $\left(K \cup M_{i-1}\right)$ $\times I$, then $C_{f^{\prime}}=C_{f}$.

It is possible to define $C_{f}$ more directly, and to prove that when $C_{f}\left(\sigma^{i}\right)=0$, the map $g: M_{\alpha} \rightarrow V_{\beta}$ appearing in part (b) of the theorem can be chosen so as to approximate $f$, and to agree with $f$ outside a given neighborhood of $M \cup K_{i}$ as well as in a neighborhood of $M \cup K_{i-1}$.

This obstruction theory can easily be modified so as to apply to piecewise regular local homeomorphisms.

5. On the groups $\Gamma_{k}$. Milnor [3] has defined a space $B_{P L}$, which is a classifying space for stable equivalence classes of piecewise linear microbundles.

Every orthogonal bundle (i.e., $n$-plane bundle with structural group $O(n)$ ) determines an underlying microbundle, hence we may consider $B_{O}$ as a subcomplex of $B_{P L}$, where $B_{O}$ is classifying space for stable equivalence classes of orthogonal bundles. Milnor conjectured that $\Gamma_{i-1} \approx \pi_{i}\left(B_{P L}, B_{0}\right)$.

THEOREM 5.1. Let $\psi$ assign to each microbundle $\xi$ over $S^{i}$ the obstruction $\psi(\xi) \in \Gamma_{i-1}$ to smoothing a regular neighborhood of the zero section of the total space of $\xi$. Then $\psi$ induces an isomorphism between $\pi_{i}\left(B_{P L}, B_{O}\right)$ and $\Gamma_{i-1}$.

Proof. We represent an element of $\pi_{i}\left(B_{P L}\right)$ by a microbundle $\xi=\left(p, E, S^{i}, j\right)$. Here $p: E \rightarrow S^{i}$ is the projection and $j: S^{i} \rightarrow E$ is the zero cross section so that $p j$ is the identity map of $S^{i}$. It is easy to prove, using 3.1, 4.2 and standard techniques of obstruction theory, that if $M$ is a $(k-1)$-connected combinatorial manifold, then there is a unique smoothing of a neighborhood of $M_{k-1}$ up to concordance, where two smoothings are identified if they agree on a common subneighborhood. Therefore the first obstruction to smoothing $M$ is a well-defined cohomology class in $H^{k}\left(M ; \Gamma_{k-1}\right)$. Moreover, $M$ and $M \times R$ have the same obstruction class. Therefore $\psi(\xi)$ is well defined, and is unchanged if a trivial bundle is added to $\xi$. It is easy to see that $\psi$ is a homomorphism.

We can identify $\xi$ with the restriction of $p^{*}(\xi)$ to $j\left(S^{i}\right)$, since $p$ is a homotopy equivalence on a regular neighborhood of $j\left(S^{i}\right)$. If $\xi$ comes from an orthogonal bundle, then $E$ can be smoothed, and so $\psi$ vanishes on the image of $\pi_{i}\left(B_{0}\right)$ in $\pi_{i}\left(B_{P L}\right)$. On the other hand, if $\psi(\xi)$ vanishes then $E$ can be smoothed, and the tangent microbundle $\tau_{E}$ of $E$ comes from an orthogonal bundle. Since $p^{*} \xi \oplus p^{*} \tau_{S^{i}}=\tau_{E}$, and 
$\tau_{S^{i}}$ is stably trivial, we see that $\xi$ comes from $\pi_{i}\left(B_{o}\right)$ if $\psi(\xi)=0$.

Using the exactness of the homotopy sequence of $\left(B_{P L}, B_{O}\right)$, we see that $\psi$ induces a monomorphism from the image of $\pi_{i}\left(B_{P L}\right)$ $\rightarrow \pi_{i}\left(B_{P L}, B_{O}\right)$ into $\Gamma_{i-1}$. It remains to prove that $\psi: \pi_{i}\left(B_{P L}\right) \rightarrow \Gamma_{i-1}$ is onto, and that $\pi_{i}\left(B_{P L}\right) \rightarrow \pi_{i}\left(B_{P L}, B_{O}\right)$ is onto. This last fact is due to Milnor, who proved it using other methods. To prove it we use exactness and show that if an orthogonal bundle $\eta$ is stably trivial as a microbundle, then $\eta$ is stably trivial as an orthogonal bundle. A neighborhood $E_{0}$ of the zero section of $\eta$ is combinatorially equivalent to $S^{i} \times R^{n}$. Give $E_{0}$ a smoothing $\alpha$, so that $\eta$ is a differential bundle. By the Product Theorem, $E_{0}$ is diffeomorphic to $S_{\alpha}^{i} \times R^{n}$ where $\alpha$ is a smoothing of $S^{i}$. Since Adams has proved that any smooth homotopy sphere is a $\pi$-manifold (cf. [1]), $E_{0}$ is parallelizable. It follows easily that $\eta$ is stably trivial.

It remains to prove that $\psi$ maps $\pi_{i}\left(B_{P L}\right)$ onto $\Gamma_{i-1}$. The following argument was suggested by Milnor, and replaces a more complicated one of the author. If $\alpha \in \Gamma_{i-1}$ is a smoothing of $S^{i-1}$, then $S_{\alpha}^{i-1} \times I$ has a trivial tangent bundle. Also $S^{i-1} \times I$ with the ordinary differential structures has a trivial tangent bundle, and the underlying microbundles are identical. This gives us a microbundle equivalence $f: S^{i-1} \times R^{i} \rightarrow S^{i-1} \times R^{i}$ which produces a microbundle $\xi$ over $S^{i}$ when two copies of $D^{i} \times R^{i}$ are glued together by $f$. It is easy to see that $\psi(\xi)=\alpha$, completing the proof of the theorem.

\section{REFERENCES}

1. J. F. Adams, Vector fields on spheres, Ann. of Math. (2) 75 (1962), 603-632.

2. M. W. Hirsch, On combinatorial submanifolds of differentiable manifolds, Comment. Math. Helv. 36 (1961), 108-111.

3. J. Milnor, Microbundles and differentiable structures, Princeton University, 1961 (mimeographed).

4. - Two complexes which are homeomorphic but combinatorially distinct, Ann. of Math. (2) 74 (1961), 575-590.

5. J. Munkres, Obstructions to the smoothing of piecerwise differentiable homeomorphisms, Ann. of Math. (2) 72 (1960), 521-554.

6. - Obstructions to imposing differentiable structures, Notices Amer. Math. Soc. 7 (1960), 204.

7. - Obstructions to extending diffeomorphisms, Princeton University, 1960 (mimeographed).

8. R. Thom, Des variêtés triangulêes aux variêtés différentiables, Proc. Internat. Congress Math., 1958, Cambridge Univ. Press, Cambridge, 1960, pp. 248-255.

University of California, Berkeley 